PROCEEDINGS OF THE

AMERICAN MATHEMATICAL SOCIETY

Volume 133, Number 10, Pages 2997-3004

S 0002-9939(05)07829-9

Article electronically published on March 22, 2005

\title{
POWER BOUNDED OPERATORS AND SUPERCYCLIC VECTORS II
}

\author{
V. MÜLLER
}

(Communicated by Joseph A. Ball)

\begin{abstract}
We show that each power bounded operator with spectral radius equal to one on a reflexive Banach space has a nonzero vector which is not supercyclic. Equivalently, the operator has a nontrivial closed invariant homogeneous subset. Moreover, the operator has a nontrivial closed invariant cone if 1 belongs to its spectrum. This generalizes the corresponding results for Hilbert space operators.

For non-reflexive Banach spaces these results remain true; however, the non-supercyclic vector (invariant cone, respectively) relates to the adjoint of the operator.
\end{abstract}

Denote by $B(X)$ the set of all bounded linear operators on a complex Banach space $X$. Let $T \in B(X)$. A vector $x \in X$ is called cyclic for $T$ if the set $\{p(T) x$ : $p$ polynomial $\}$ is dense in $X$. The vector $x$ is called hypercyclic if the set $\left\{T^{n} x\right.$ : $n=0,1, \ldots\}$ is dense in $X$. Clearly $T$ has a nontrivial (closed) invariant subspace if and only if there is a nonzero vector which is not cyclic for $T$. Similarly, $T$ has a nontrivial closed invariant subset if and only if there is a nonzero vector which is not hypercyclic. Thus the notions of cyclic and hypercyclic vectors are closely connected with the invariant subspace/subset problem.

By the well-known example of Read $[\underline{R}]$, there is an operator (acting on $\ell_{1}$ ) without nontrivial closed invariant subsets. Equivalently, every nonzero vector is hypercyclic. For operators on Hilbert spaces (and more generally on reflexive Banach spaces) the invariant subspace/subset problem is still open. For Hilbert and reflexive spaces there are rather some partial positive results indicating that the reflexivity of the space might play an important role in these problems.

By $[\mathrm{BCP}$, every Hilbert space contraction whose spectrum contains the unit circle has a nontrivial invariant subspace. A Banach space version of this result was proved in [AM]: a polynomially bounded operator on a reflexive Banach space whose spectrum contains the unit circle has a nontrivial invariant subspace.

An operator $T \in B(X)$ is called power bounded if $\sup _{n}\left\|T^{n}\right\|<\infty$. Clearly a power bounded operator has no hypercyclic vectors since all orbits $\left\{T^{n} x: n=\right.$ $0,1, \ldots\}$ are bounded. However, this argument is not valid if we replace hypercyclicity by supercyclicity.

Received by the editors April 15, 2004 and, in revised form, May 17, 2004.

2000 Mathematics Subject Classification. Primary 47A16, 47A15.

Key words and phrases. Supercyclic vectors, invariant subspace problem, positive operators, power bounded operators.

This research was supported by grant No. 201/03/0041 of GA ČR. 
A vector $x \in X$ is called supercyclic for an operator $T \in B(X)$ if the set $\left\{\lambda T^{n} x\right.$ : $\lambda \in \mathbb{C}, n=0,1, \ldots\}$ is dense in $X$. The concept of supercyclicity lies between the cyclic and hypercyclic vectors.

In [M2], the following analogue of the result $\mathrm{BCP}$ ] was proved: if $T$ is a power bounded operator on a Hilbert space with spectral radius equal to 1, then there is a nonzero vector which is not supercyclic for $T$. Equivalently, there is a closed $T$-invariant subset $M$ which is homogeneous (i.e., $\mathbb{C} M \subset M$ ).

The aim of this paper is to generalize this result for reflexive Banach spaces. Moreover, if $1 \in \sigma(T)$, then $T$ has a nontrivial closed invariant cone (i.e., a subset $L$ satisfying $L+L \subset L$ and $t L \subset L$ for all $t \geq 0$ ).

For non-reflexive Banach spaces these results remain true, but the constructed non-supercyclic vector (resp. invariant cone) is for $T^{*}$ instead of $T$.

For an operator $T \in B(X)$ denote by $\sigma(T)$ and $r(T)$ its spectrum and spectral radius, respectively. Let $\sigma_{e}(T)$ be the essential spectrum of $T, \sigma_{e}(T)=\{\lambda \in \mathbb{C}$ : $T-\lambda$ is not Fredholm $\}$.

For a subspace $L \subset X$ denote by $L^{\perp}$ its annihilator,

$$
L^{\perp}=\left\{y \in X^{*}:\langle x, y\rangle=0 \text { for all } x \in L\right\} .
$$

Similarly, for $M \subset X^{*}$ denote by ${ }^{\perp} M$ the pre-annihilator,

$$
{ }^{\perp} M=\{x \in X:\langle x, y\rangle=0 \text { for all } y \in M\} .
$$

Theorem 1. Let $X$ be a Banach space which contains no isomorphic copy of $c_{0}$. Let $T \in B(X)$ satisfy that $1 \in \sigma(T)$ and $T^{n} x \rightarrow 0$ for all $x \in X$. Let $\left(a_{n}\right)_{n=0}^{\infty}$ be a sequence of positive numbers such that $\lim _{n \rightarrow \infty} a_{n}=0$. Then there exist vectors $x \in X$ and $y \in X^{*}$ such that $\operatorname{Re}\left\langle T^{n} x, y\right\rangle>a_{n}$ for all $n \geq 0$.

Moreover, if there is a Banach space $Y$ such that $X$ is a (isometrical) subspace of $Y^{*}$, then the vector $y$ can be chosen in $Y$.

Proof. By the Banach-Steinhaus theorem, the operator $T$ is power bounded. Let $K=\sup _{n}\left\|T^{n}\right\|$. Clearly $r(T)=1$.

Replacing the numbers $a_{n}$ by $\sup \left\{a_{i}: i \geq n\right\}$ we can assume without loss of generality that $a_{0} \geq a_{1} \geq a_{2} \geq \cdots$.

Suppose first that $1 \notin \sigma_{e}(T)$. Then 1 is an eigenvalue of $T$ and there exists a nonzero vector $x \in X$ such that $T x=x$. Let $y \in X^{*}$ satisfy $\operatorname{Re}\langle x, y\rangle>\sup _{n} a_{n}$. Then $\operatorname{Re}\left\langle T^{n} x, y\right\rangle=\operatorname{Re}\langle x, y\rangle>a_{n}$ for all $n$. In the second case $\left(X \subset Y^{*}\right)$ we can choose $y \in Y$ with the same properties.

Therefore we can assume that $1 \in \sigma_{e}(T)$. Thus $1 \in \partial \sigma_{e}(T)$ and, by [HW], $T-I$ is not upper semi-Fredholm. Consequently, if $\varepsilon>0$ and $M \subset X$ is a subspace of finite codimension, then there exists $u \in M$ of norm one such that $\|T u-u\|<\varepsilon$. Moreover, given $n_{0} \in \mathbb{N}$, we can also find $w \in M$ of norm one such that $\left\|T^{j} w-w\right\|<$ $\varepsilon$ for all $j \leq n_{0}$.

We first prove the following auxiliary statement:

Claim A. Let $F, M$ be subspaces of $X, \operatorname{dim} F<\infty, \operatorname{codim} M<\infty, \varepsilon>0$, let $v_{1}, \ldots, v_{m}$ be a finite number of elements of $X^{*}$ (of $Y$, respectively), and let 
$r, r^{\prime} \in \mathbb{N}$. Then there exist $u \in M,\|u\|=1$ and $r^{\prime \prime} \in \mathbb{N}, r^{\prime \prime}>\max \left\{r, r^{\prime}\right\}$ such that

$$
\begin{aligned}
& \left\|T^{j} u-u\right\| \leq \varepsilon \quad(j \leq r), \\
& \left\|T^{j} u\right\| \leq \varepsilon \quad\left(j \geq r^{\prime \prime}\right), \\
& \left|\left\langle T^{j} u, v_{l}\right\rangle\right| \leq \varepsilon(K+1)\left\|v_{l}\right\| \quad\left(l=1, \ldots, m, r \leq j<r^{\prime \prime}\right), \\
& \operatorname{dist}\{u, F\}>\frac{1}{3} .
\end{aligned}
$$

Proof. We construct inductively vectors $x_{1}, x_{2}, \cdots \in X$ of norm one and an increasing sequence of integers $n_{1}<n_{2}<\cdots$ such that

$$
\begin{aligned}
& \left\|T^{j} x_{k}-x_{k}\right\|<2^{-k} \quad\left(j \leq n_{k}\right), \\
& \left\|T^{j} x_{i}\right\|<2^{-i} \quad\left(j \geq n_{k}, i<k\right), \\
& \left\|\sum_{i=1}^{\infty} \alpha_{i} x_{i}\right\| \geq \frac{1}{3}\left\|\sum_{t}^{t^{\prime}} \alpha_{i} x_{i}\right\| \quad\left(\alpha_{i} \in \mathbb{C}, t \leq t^{\prime}\right) .
\end{aligned}
$$

Let $k \geq 1$ and suppose that the vectors $x_{1}, \ldots, x_{k-1} \in X$ and numbers $n_{1}<n_{2}<$ $\cdots<n_{k-1}$ have already been constructed. Since $T^{n} x \rightarrow 0$ for all $x \in X$, we can choose $n_{k}>n_{k-1}$ such that (6) is satisfied (for $k=1$ set $n_{1}=r$ ).

Let $F_{k}=F \vee\left\{x_{1}, \ldots, x_{k-1}\right\}$. By [M1], there exists a subspace $M_{k} \subset X$ of finite codimension such that

$$
\|f+g\| \geq \frac{5}{6} \max \{\|f\|,\|g\| / 2\}
$$

for all $f \in F_{k}$ and $g \in M_{k}$. Set

$$
M_{k}^{\prime}=M \cap \bigcap_{i=1}^{k} M_{i} \cap\left\{x \in X:\left\langle T^{j} x, v_{l}\right\rangle=0 \quad\left(l=1, \ldots, m, j \leq n_{k}\right)\right\} .
$$

Since $1 \in \partial \sigma_{e}(T)$ and $\operatorname{codim} M_{k}^{\prime}<\infty$, there exists $x_{k} \in M_{k}^{\prime} \subset M$ of norm one satisfying (5).

Let $\left(x_{i}\right)_{i=1}^{\infty}$ and $\left(n_{i}\right)_{i=1}^{\infty}$ be the sequences constructed in the above-described way. Clearly they satisfy conditions (5) and (6). Let $\alpha_{i} \in \mathbb{C}, 1 \leq t \leq t^{\prime}<\infty$ and suppose that the sum $\sum_{i=1}^{\infty} \alpha_{i} x_{i}$ converges. Since $\sum_{i=1}^{t^{\prime}} \alpha_{i} x_{i} \in F_{t^{\prime}+1}, \sum_{i=t^{\prime}+1}^{\infty} \alpha_{i} x_{i} \in M_{t^{\prime}+1}$, $\sum_{i=1}^{t-1} \alpha_{i} x_{i} \in F_{t}$ and $\sum_{i=t}^{t^{\prime}} \alpha_{i} x_{i} \in M_{t}$, we have

$$
\left\|\sum_{i=1}^{\infty} \alpha_{i} x_{i}\right\| \geq \frac{5}{6}\left\|\sum_{i=1}^{t^{\prime}} \alpha_{i} x_{i}\right\| \geq \frac{1}{2} \cdot\left(\frac{5}{6}\right)^{2}\left\|\sum_{i=t}^{t^{\prime}} \alpha_{i} x_{i}\right\| \geq \frac{1}{3}\left\|\sum_{i=t}^{t^{\prime}} \alpha_{i} x_{i}\right\| .
$$

In particular, $\left\|\sum_{i} \alpha_{i} x_{i}\right\| \geq \frac{1}{3} \max _{i}\left|\alpha_{i}\right|$ whenever the sum $\sum_{i} \alpha_{i} x_{i}$ converges.

Since $X$ contains no isomorphic copy of $c_{0}$, we can find $s \in \mathbb{N}$ and a finite linear combination $u=\sum_{i=1}^{s} \alpha_{i} x_{i}$ such that $\|u\|=1$ and $\max \left\{\left|\alpha_{i}\right|: 1 \leq i<s\right\} \leq \varepsilon$. Clearly $u \in M$. Without loss of generality, we can assume that $n_{s+1}>r^{\prime}$. Set $r^{\prime \prime}=n_{s+1}$.

If $j \leq r=n_{1}$, then

$$
\left\|T^{j} u-u\right\|=\left\|\sum_{i=1}^{s} \alpha_{i}\left(T^{j} x_{i}-x_{i}\right)\right\| \leq \varepsilon \sum_{i=1}^{s}\left\|T^{j} x_{i}-x_{i}\right\| \leq \varepsilon \sum_{i=1}^{s} 2^{-i} \leq \varepsilon .
$$


If $j \geq r^{\prime \prime}$, then similarly

$$
\left\|T^{j} u\right\|=\left\|\sum_{i=1}^{s} \alpha_{i} T^{j} x_{i}\right\| \| \leq \varepsilon \sum_{i=1}^{s} 2^{-i} \leq \varepsilon .
$$

To show (3), let $1 \leq l \leq m, 1 \leq k \leq s$ and $n_{k} \leq j<n_{k+1}$. Then

$$
\begin{aligned}
& \left|\left\langle T^{j} u, v_{l}\right\rangle\right| \leq\left|\left\langle\sum_{i=1}^{k-1} \alpha_{i} T^{j} x_{i}, v_{l}\right\rangle\right|+\left|\left\langle\alpha_{k} T^{j} x_{k}, v_{l}\right\rangle\right|+\left|\left\langle\sum_{i=k+1}^{s} \alpha_{i} T^{j} x_{i}, v_{l}\right\rangle\right| \\
& \leq \varepsilon \sum_{i=1}^{k-1} 2^{-i}\left\|v_{l}\right\|+\varepsilon K\left\|v_{l}\right\| \leq \varepsilon(K+1)\left\|v_{l}\right\| .
\end{aligned}
$$

Since $u \in M_{1}$ and $F \subset F_{1}$, we have

$$
\operatorname{dist}\{u, F\}=\inf \{\|u+f\|: f \in F\} \geq \frac{5}{12}\|u\|>\frac{1}{3} .
$$

This finishes the proof of Claim A.

Continuation of the proof of Theorem 1. By Lemma 4 of [M2], there are positive numbers $\beta_{m}(m=1,2, \ldots)$ such that $\sum_{i=1}^{\infty} \beta_{i}<\frac{1}{3}$ and

$$
\sum_{i=m+1}^{\infty} \beta_{i}^{2}>3 K \beta_{m}^{2}
$$

for all $m \geq 1$.

For $m=1,2, \ldots$ choose inductively positive numbers $\varepsilon_{m}$ such that $\varepsilon_{1}>\varepsilon_{2}>\ldots$ and

$$
\sum_{i=m+1}^{\infty} \beta_{i}^{2}-3 K \beta_{m}^{2}>(K+2) \varepsilon_{m} .
$$

Using Claim A we construct inductively an increasing sequence $r_{1}<r_{2}<\ldots$ of positive integers and vectors $u_{m} \in X$ and $v_{m} \in X^{*}\left(v_{m} \in Y\right.$, respectively) such that $\left\|u_{m}\right\|=1,\left\|v_{m}\right\| \leq 3,\left\langle u_{m}, v_{m}\right\rangle=1$ for all $m=1,2, \ldots$, and

$$
\begin{aligned}
& \sum_{i=m+1}^{\infty} \beta_{i}^{2}-3 K \beta_{m}^{2}-(K+2) \varepsilon_{m}>a_{r_{m}}, \\
& \left\|T^{j} u_{m}-u_{m}\right\| \leq \varepsilon_{m} \quad\left(j \leq r_{m}\right), \\
& \left\|T^{j} u_{s}\right\| \leq \varepsilon_{m+1} \quad\left(j \geq r_{m+1}, s \leq m\right), \\
& \left|\left\langle T^{j} u_{m}, v_{s}\right\rangle\right| \leq 3 \varepsilon_{m}(K+1) \quad\left(m, s \in \mathbb{N}, s<m, r_{m} \leq j<r_{m+1}\right), \\
& \left\langle u_{m}, v_{s}\right\rangle=0 \quad(s<m), \\
& \left|\left\langle T^{j} u_{s}, v_{m}\right\rangle\right|=0 \quad\left(m, s \in \mathbb{N}, s<m, j \leq r_{m}\right) .
\end{aligned}
$$

Choose $r_{1}$ such that $\sum_{i=2}^{\infty} \beta_{i}^{2}-3 K \beta_{1}^{2}-(K+2) \varepsilon_{1}>a_{r_{1}}$.

Suppose that $m \geq 1$ and the numbers $r_{1}<r_{2}<\cdots<r_{m}$, vectors $u_{1}, \ldots, u_{m-1} \in$ $X$ and $v_{1}, \ldots, v_{m-1} \in X^{*}$ (resp., $v_{1}, \ldots, v_{m-1} \in Y$ ) satisfying conditions (8)-(13) have already been constructed. Find $r^{\prime}$ such that

$$
\sum_{i=m+2}^{\infty} \beta_{i}^{2}-3 K \beta_{m+1}^{2}-(K+2) \varepsilon_{m+1}>a_{r^{\prime}}
$$


and

$$
\left\|T^{j} u_{s}\right\| \leq \varepsilon_{m+1} \quad\left(s \leq m-1, j \geq r^{\prime}\right)
$$

Let $F=\bigvee\left\{T^{j} u_{s}: 1 \leq s \leq m-1,0 \leq j \leq r_{m}\right\}$ and $M=\left\{x \in X:\left\langle x, v_{s}\right\rangle=\right.$ $0(s=1, \ldots, m-1)\}$. By Claim A for the numbers $\varepsilon=\varepsilon_{m}, r=r_{m}$ and the vectors $v_{1}, \ldots, v_{m-1}$, we can find an integer $r_{m+1}>\max \left\{r_{m}, r^{\prime}\right\}$ and a vector $u_{m} \in M$ such that $\left\|u_{m}\right\|=1$, $\operatorname{dist}\left\{u_{m}, F\right\}>\frac{1}{3}$ and the conditions (8)-(12) are satisfied.

Since $\operatorname{dist}\left\{u_{m}, F\right\}>\frac{1}{3}$, by the Hahn-Banach theorem we can find a functional $v_{m} \in F^{\perp}$ such that $\left\langle u_{m}, v_{m}\right\rangle=1$ and $\left\|v_{m}\right\| \leq 3$.

In the second case $\left(X \subset Y^{*}\right)$, we have $\left({ }^{\perp} F\right)^{*}=Y^{*} /\left({ }^{\perp} F\right)^{\perp}=Y^{*} / F$. Since $\left\|u_{m}+F\right\|_{Y^{*} / F}=\operatorname{dist}\left\{u_{m}, F\right\}>1 / 3$, there exists $v_{m} \in{ }^{\perp} F$ such that $\left\|v_{m}\right\| \leq 3$, $\left\langle u_{m}, v_{m}\right\rangle=1$. Thus in both cases we have (13).

Let $\left(u_{i}\right)_{i=1}^{\infty}$ and $\left(v_{i}\right)_{i=1}^{\infty}$ be the sequences constructed in the above-described way.

Set $u=\sum_{i=1}^{\infty} \beta_{i} u_{i}$ and $v=\sum_{i=1}^{\infty} \beta_{i} v_{i}$. Then $\|u\| \leq 1$ and $\|v\| \leq 1$.

Let $m \in \mathbb{N}, r_{m} \leq j<r_{m+1}$. Then

$$
\begin{gathered}
\operatorname{Re}\left\langle T^{j} u, v\right\rangle=\operatorname{Re}\left\langle\sum_{i=1}^{m-1} \beta_{i} T^{j} u_{i}, v\right\rangle+\beta_{m} \operatorname{Re}\left\langle T^{j} u_{m}, v\right\rangle+\operatorname{Re}\left\langle\sum_{i=m+1}^{\infty} \beta_{i} T^{j} u_{i}, v\right\rangle \\
\geq-\sum_{i=1}^{m-1} \beta_{i} \varepsilon_{m}+\operatorname{Re} \sum_{i=1}^{m-1} \beta_{i} \beta_{m}\left\langle T^{j} u_{m}, v_{i}\right\rangle+\beta_{m}^{2} \operatorname{Re}\left\langle T^{j} u_{m}, v_{m}\right\rangle \\
+\operatorname{Re}\left\langle\sum_{i=m+1}^{\infty} \beta_{i}\left(T^{j} u_{i}-u_{i}\right), v\right\rangle+\operatorname{Re}\left\langle\sum_{i=m+1}^{\infty} \beta_{i} u_{i}, v\right\rangle \\
\geq-\sum_{i \neq m} \beta_{i} \varepsilon_{m}-3 \varepsilon_{m} \beta_{m}(K+1) \sum_{i=1}^{m-1} \beta_{i}-3 K \beta_{m}^{2}+\sum_{i=m+1}^{\infty} \beta_{i}^{2} \\
\geq \sum_{i=m+1}^{\infty} \beta_{i}^{2}-3 K \beta_{m}^{2}-\varepsilon_{m}(K+2) \geq a_{r_{m}} \geq a_{j} .
\end{gathered}
$$

For $j<r_{1}$ we have

$$
\begin{aligned}
& \operatorname{Re}\left\langle T^{j} u, v\right\rangle=\operatorname{Re}\left\langle\sum_{i=1}^{\infty} \beta_{i} T^{j} u_{i}, v\right\rangle \geq \operatorname{Re}\left\langle\sum_{i=1}^{\infty} \beta_{i} u_{i}, v\right\rangle-\sum_{i=1}^{\infty} \beta_{i}\left\|T^{j} u_{i}-u_{i}\right\| \\
& \geq \sum_{i=1}^{\infty} \beta_{i}^{2}-\sum_{i=1}^{\infty} \beta_{i} \varepsilon_{i} \geq \sum_{i=1}^{\infty} \beta_{i}^{2}-\varepsilon_{1}>0 .
\end{aligned}
$$

Thus for suitable multiples $x$ and $y$ of $u$ and $v$, respectively, we have the statement of Theorem 1.

The following corollary improves the results of [N] and [M3].

Corollary 2. Let $X$ be a reflexive Banach space, let $T \in B(X)$ be a power bounded operator satisfying $r(T)=1$ and $T^{n} x \rightarrow 0$ for all $x \in X$. Let $\left(a_{n}\right)$ be a sequence of positive numbers satisfying $\lim _{n} a_{n}=0$. Then there exist $v \in X$ and $v^{*} \in X^{*}$ such that

$$
\left|\left\langle T^{j} v, v^{*}\right\rangle\right| \geq a_{j}
$$

for all $j \geq 1$. 
Proof. Note that $X$ contains no isomorphic copy of $c_{0}$. Since $r(T)=1$, there exists $\lambda \in \sigma(T)$ with $|\lambda|=1$. Thus the operator $S=\lambda^{-1} T$ satisfies all the conditions required in Theorem 1, which implies the statement of Corollary 2.

A subset $M$ of a Banach space $X$ is called a cone if $M+M \subset M$ and $t M \subset M$ for all $t \geq 0$.

Theorem 3. Let $X$ be a Banach space, and let $T \in B(X)$ be a power bounded operator satisfying $1 \in \sigma(T)$. Then there are nonzero vectors $v^{*} \in X^{*}$ and $v^{* *} \in$ $X^{* *}$ such that

$$
\operatorname{Re}\left\langle T^{* j} v^{*}, v^{* *}\right\rangle \geq 0
$$

for all $j \geq 0$. Consequently, $T^{*}$ has a nontrivial closed invariant cone.

Proof. The statement of Theorem 3 is clear if $T^{*}$ has a nontrivial invariant subspace. Indeed, if $M^{\prime} \subset X^{*}$ is a nontrivial subspace invariant with respect to $T^{*}$, then for any nonzero vectors $v^{*} \in M^{\prime}$ and $v^{* *} \in M^{\prime \perp}$ we have $\left\langle T^{* j} v^{*}, v^{* *}\right\rangle=0$ for all $j$.

Therefore we can assume without loss of generality that $X^{*}$ is separable (otherwise for any nonzero $x^{*} \in X^{*}$ the vectors $T^{* j} x^{*}$ generate a nontrivial subspace invariant with respect to $T^{*}$ ). By a result of Bessaga and Pełczynski (see [DU] p. 83]), $X^{*}$ contains no isomorphic copy of $c_{0}$.

We may also assume that $T$ is not a scalar multiple of the identity operator, since the statement becomes trivial in this case.

We use the standard reduction technique; see $\left[\mathrm{NF}\right.$. Set $M_{1}=\left\{x^{*} \in X^{*}\right.$ : $\left.T^{* n} x \rightarrow 0\right\}$. Then $M_{1}$ is a closed subspace invariant with respect to $T^{*}$. If $M_{1}$ is nontrivial, then Theorem 3 is proved. If $M_{1}=X^{*}$, then the statement follows from Theorem 1. Thus we may assume that $M_{1}=\{0\}$.

Let $M=\left\{x \in X: T^{n} x \rightarrow 0\right\}$. Then $M$ is a closed subspace invariant with respect to $T$, and so $M^{\perp}$ is invariant with respect to $T^{*}$. If $M$ is nontrivial, then $M^{\perp}$ is nontrivial, and so Theorem 3 is proved. If $M=\{0\}$, then $T$ is a power bounded operator of class $C_{11}$, and so $T^{*}$ has plenty of invariant subspaces by [CF] p. 136] and [AM]. Therefore we may assume that $M=X$.

Let $M_{2}=\left\{x^{* *} \in X^{* *}: T^{* * n} x \rightarrow 0\right\}$. Consider the operator $S=T^{* *} \mid M_{2}$. Clearly $X \subset M_{2}$ and $S$ is an extension of $T$. Since $1 \in \partial \sigma(T) \subset \sigma_{\pi}(T)$, where $\sigma_{\pi}$ denotes the approximate point spectrum, we have $1 \in \sigma(S)$. Thus the second statement of Theorem 1 for the operator $S$ gives the existence of $v^{* *} \in M_{2} \subset X^{* *}$ and $v^{*} \in X^{*}$ satisfying the statement of Theorem 3 .

Thus in all cases there are nonzero vectors $v^{*} \in X^{*}$ and $v^{* *} \in X^{* *}$ satisfying $\operatorname{Re}\left\langle T^{* j} v^{*}, v^{* *}\right\rangle \geq 0$ for all $j$. Then the closed cone generated by the vectors $T^{* j} v^{*}(j=0,1, \ldots)$ is nontrivial and invariant with respect to $T^{*}$.

In fact, the set $\left\{x^{*} \in X^{*}: \operatorname{Re}\left\langle x^{*}, T^{* * j} v^{* *}\right\rangle \geq 0\right.$ for all $\left.j\right\}$ is another nontrivial closed cone invariant with respect to $T^{*}$.

Corollary 4. Let $X$ be a reflexive Banach space, and let $T \in B(X)$ be a power bounded operator satisfying $1 \in \sigma(T)$. Then there are vectors $v \in X$ and $v^{*} \in X^{*}$ such that

$$
\operatorname{Re}\left\langle T^{j} v, v^{*}\right\rangle \geq 0
$$

for all $n \geq 0$. In particular, $T$ has a nontrivial closed invariant cone.

Remark 5. (1) In [M2], it was wrongly stated that each power bounded Hilbert space operator $T$ satisfying $r(T)=1$ has a nontrivial closed invariant cone. In fact the result was proved only under the assumption that $1 \in \sigma(T)$. 
(2) Let $L \subset X$ be a cone satisfying the condition

$$
L \cap(-L)=\{0\}
$$

(in fact this condition is sometimes included in the definition of a cone).

A cone $L$ satisfying (14) defines naturally a partial order on $X$ so that the space $X$ becomes an ordered vector space. An operator $T \in B(X)$ satisfying $T L \subset L$ is called positive.

The invariant cone constructed in Theorem 4 in general does not satisfy (14). However, if (14) is not satisfied, then $L \cap(-L)$ is a nontrivial closed real subspace invariant with respect to $T$ (a subset $M \subset X$ is called a real subspace if $M+M \subset M$ and $t M \subset M$ for all real $t)$.

Thus we have the following alternative:

Theorem 6. Let $X$ be a reflexive Banach space, and let $T \in B(X)$ be a power bounded operator satisfying $1 \in \sigma(T)$. Then either $T$ has a nontrivial invariant real subspace, or there is a partial order $\leq$ on $X$ such that $X$ becomes an ordered vector space and $T$ a positive operator. Moreover, the cone $\{x \in X: x \geq 0\}$ is closed.

It is a well-known generalization of the Perron-Frobenius theorem that each positive operator on a Banach lattice satisfies $r(T) \in \sigma(T)$. In fact the condition in Theorem 1 implies this statement too (provided that the numbers $a_{j}$ are decreasing sufficiently slowly).

Proposition 7. Let $T \in B(X), r(T) \leq 1$, let $x \in X, x^{*} \in X^{*}$ and let $\operatorname{Re}\left\langle T^{j} x, x^{*}\right\rangle$ $\geq(j+1)^{-1}$ for all $j$. Then $1 \in \sigma(T)$.

Proof. For $z \in \mathbb{C},|z|>1$ we have $(z-T)^{-1}=\sum_{i=0}^{\infty} \frac{T^{j}}{z^{j+1}}$. Therefore

$$
\begin{aligned}
& \limsup _{t \rightarrow 1_{+}}\left\|(t-T)^{-1}\right\| \geq \frac{1}{\|x\| \cdot\left\|x^{*}\right\|} \limsup _{t \rightarrow 1_{+}}\left|\left\langle(t-T)^{-1} x, x^{*}\right\rangle\right| \\
& \geq \frac{1}{\|x\| \cdot\left\|x^{*}\right\|} \limsup _{t \rightarrow 1_{+}} \operatorname{Re}\left\langle\sum_{j=0}^{\infty} \frac{T^{j} x}{t^{j+1}}, x^{*}\right\rangle \geq \frac{1}{\|x\| \cdot\left\|x^{*}\right\|} \limsup _{t \rightarrow 1_{+}} \sum_{j=0}^{\infty} \frac{1}{(j+1) t^{j+1}} .
\end{aligned}
$$

By an elementary calculation it is possible to show that the last expression is equal to $\infty$, and so the resolvent $(z-T)^{-1}$ is unbounded in each neighbourhood of 1 . Therefore $1 \in \sigma(T)$.

Theorem 8. Let $X$ be a reflexive Banach space, $\operatorname{dim} X \geq 2$, and let $T \in B(X)$ be a power bounded operator satisfying $r(T)=1$. Then there is a nonzero vector $v \in X$ which is not supercyclic for $T$.

Proof. Without loss of generality we may assume that $1 \in \sigma(T)$ and that $T$ is not a scalar multiple of the identity operator. We may also assume that the point spectrum of $T^{*}$ is empty (otherwise, if $\lambda$ is an eigenvalue of $T^{*}$, then $\overline{(T-\lambda) X}$ is a nontrivial subspace invariant with respect to $T$ ).

Let $v \in X, v^{*} \in X^{*}$ be nonzero vectors satisfying $\operatorname{Re}\left\langle T^{j} v, v^{*}\right\rangle \geq 0$ for all $j$, which were constructed in Corollary 4 . Then

$$
\left\{t T^{j} v: t \geq 0, j=0,1, \ldots\right\}^{-} \subset\left\{x \in X: \operatorname{Re}\left\langle x, v^{*}\right\rangle \geq 0\right\} \neq X .
$$

By [LM], $v$ is not supercyclic for $T$. 
Corollary 9. Let $X$ be a reflexive Banach space, $\operatorname{dim} X \geq 2$, and let $T \in B(X)$ satisfy $\|T\|=r(T)$. Then there is a nonzero vector $v \in X$ which is not supercyclic for $T$.

Proof. The statement is clear if $T=0$. If $T \neq 0$, then consider the contraction $T /\|T\|$.

Clearly Theorem 8 and Corollary 9 remain true for non-reflexive Banach spaces. However, in this case the non-supercyclic vector exists for $T^{*}$ instead of $T$.

\section{REFERENCES}

[AM] C. Ambrozie, V. Müller, Invariant subspaces for polynomially bounded operators, J. Funct. Anal. (to appear).

[BCP] S. Brown, B. Chevreau, C. Pearcy., On the structure of contraction operators II, J. Funct. Anal. 76 (1988), 30-5 5. MR0923043 (90b:47030b)

[CF] I. Colojoară, C. Foiaş, Theory of Generalized Spectral Operators, Gordon and Breach, New York London Paris, 1968. MR0394282 (52:15085)

[DU] J. Diestel, J.J. Uhl, Jr., Vector Measures, Mathematical Surveys 15, Ame rican Mathematical Society, Providence, Rhode Island, 1977. MR0453964 (56:12216)

[HW] R. Harte, A. Wickstead, Upper and lower Fredholm spectra II, Math. Z. 154 (1977), 253256. MR $0454677(56: 12926)$

[LM] F. Leon, V. Müller, Rotations of hypercyclic operators, Int. Eq. Operator Theory (to appear).

[M1] V. Müller, Local behaviour of the polynomial calculus of operators, J. reine angew. Math. 430 (1992), 61-68. MR1172907|(94b:47004)

[M2] V. Müller, Power bounded operators and supercyclic vectors, Proc. Amer. Math. Soc. 131 (2003), 3807-3812. MR 1999927 (2004e:47011)

[M3] - Orbits, weak orbits and local capacity of operators, Integral Equations Operator Theory 41 (2000), 230-253. MR 1847174|(2002g:47009)

[NF] B. Sz.-Nagy, C. Foiaş, Harmonic Analysis of Operators, Akadémiai Kiadó/North Holland, Budapest/Amsterdam, 1970. MR0275190 (43:947)

[N] J.M.A.M. van Neerven, On the orbits of an operator with spectral radius one, Czechoslovak Math. J. 45 (1995), 495 -502. MR1344516|(96f:47009)

[R] C.J. Read, The invariant subspace problem for a class of Banach spaces II. Hypercyclic operators, Israel J. Math. 63 (1988), 1-40. MR0959046 (90b:47013)

Mathematical Institute, Czech Academy of Sciences, Žitná 25, 11567 Prague 1, Czech REPUBLIC

E-mail address: muller@math.cas.cz 